\title{
La bitácora como estrategia didáctica en el curso de Introducción a la Pedagogía de la Universidad de Costa Rica durante la crisis del COVID-19
}

\author{
The blog as a didactic strategy in the Introduction to Pedagogy course \\ at the University of Costa Rica during the COVID-19 crisis \\ O blog como estratégia de ensino no curso de Introdução à Pedagogia \\ da Universidade da Costa Rica durante a crise da COVID-19
}

Juan Carlos Naranjo Segura
Universidad de Costa Rica
San José, Costa Rica

Recibido - Received - Recebido: 14 / 07 / 2020 Corregido - Revised - Revisado: 21 / 08 / 2020 Aceptado - Accepted - Aprovado: 01 / 09 / 2020

DOI: https://doi.org/10.22458/ie.v22iespecial..3147

URL: https://revistas.uned.ac.cr/index.php/innovaciones/article/view/3147

\begin{abstract}
Resumen: El artículo expone un diagnóstico sobre los aportes y limitaciones de la bitácora como estrategia didáctica durante la crisis del COVID-19, para su valoración en el alcance de los aprendizajes en la población estudiantil durante el desarrollo del curso Introducción a la Pedagogía, perteneciente a la Escuela de Formación Docente de la Universidad de Costa Rica, impartido el primer semestre 2020. Se trabajó con cuatro grupos de estudiantes del curso y dos de sus docentes. La metodología utilizada fue desde el enfoque cualitativo porque se buscaba evidenciar las experiencias y puntos de vista de dichos actores, para ello se empleó la entrevista y el cuestionario cualitativo con preguntas abiertas y cerradas para el logro del objetivo de la investigación. Dentro de los principales resultados se obtuvo el reconocimiento de que la bitácora es una estrategia didáctica útil para el desarrollo del curso en medio de la crisis provocada por la pandemia. La bitácora fomentó la capacidad de síntesis y el desarrollo de un conocimiento integrador, el alcance de un aprendizaje significativo y útil para el grupo de estudiantes, el desarrollo del autoaprendizaje, la retroalimentación docente en un contexto de virtualidad, la flexibilización de los procesos de aprendizajes según las necesidades y realidades de estudiantes y docentes. Las principales limitaciones fueron que su uso requirió mucho tiempo y evidenció un desgaste a lo largo del semestre, así como el acceso a Internet.
\end{abstract}

Palabras clave: Bitácora, estrategia didáctica, aporte, limitación, COVID-19, educación

\begin{abstract}
The article presents a diagnosis on the contributions and limitations of the logbook as a didactic strategy during the COVID-19 crisis. It aims to assess this within the scope of learning in the student population during the development of the Introduction to Pedagogy course, belonging to the School of Teacher Training of the University of Costa Rica, taught in the first semester of 2020. We worked with four groups of students of the course and two of their teachers. The methodology used was from the qualitative approach because it sought to show the experiences and points of view of said actors. For this, the interview and the qualitative questionnaire with open and closed questions were used to achieve the research goal. Among the main results, there was the recognition obtained that the logbook is a useful didactic strategy for the development of the course in the midst of the crisis caused by the pandemic. The logbook encouraged the capacity for synthesis and the development of integrating knowledge, the achievement of meaningful and useful learning for the group of students, the development of self-learning, teacher feedback in a virtual context, the flexibility of learning
\end{abstract}


processes according to the needs and realities of students and teachers. The main limitations were first, that its use took a long time and weariness was evident throughout the semester, and second the Internet access.

Key Words: Blog, didactic strategy, contribution, limitation, COVID-19, education

Resumo: $\bigcirc$ artigo apresenta um diagnóstico sobre as contribuições e limitações do livro de registro como estratégia de ensino durante a crise da COVID-19, para avaliar o alcance do aprendizado na população estudantil durante o desenvolvimento do curso Introdução à Pedagogia, pertencente à Escola de Formação de Professores da Universidade da Costa Rica, lecionou no primeiro semestre de 2020. Trabalhamos com quatro grupos de alunos do curso e dois de seus professores. A metodologia utilizada foi a partir da abordagem qualitativa porque buscou destacar as experiências e pontos de vista desses atores, utilizando a entrevista e o questionário qualitativo com perguntas abertas e fechadas para alcançar o objetivo da pesquisa. Entre os principais resultados, foi reconhecido que o livro de bordo é uma estratégia de ensino útil para o desenvolvimento do curso em meio à crise provocada pela pandemia. O blog promoveu a capacidade de síntese e o desenvolvimento de um conhecimento integrador, o alcance de uma aprendizagem significativa e útil para o grupo de estudantes, o desenvolvimento da autoaprendizagem, o feedback dos professores em um contexto de virtualidade, a flexibilidade dos processos de aprendizagem de acordo com as necessidades e realidades dos estudantes e professores. As principais limitações foram que seu uso exigiu muito tempo e mostrou desgaste durante todo o semestre, assim como o acesso à Internet.

Palavras-chave: Revista, estratégia de ensino, contribuição, limitação, COVID-19, educação

\section{INTRODUCCIÓN}

La llegada del ocaso del año 2019 anunciaba el inicio de una nueva enfermedad que atentaba contra la salud de la humanidad, la enfermedad es el coronavirus de tipo 2 causante del síndrome respiratorio agudo severo (SRAS-CoV-2), dado a conocer por la Organización Mundial de la Salud a partir de febrero del 2020 como la COVID-19. Su expansión en pocos meses se dio a escala planetaria, convirtiéndose en pandemia, y con ella, sus múltiples secuelas en todos los ámbitos de la sociedad mundial. Uno de estos sectores es el de educación.

Ante tal escenario, el sistema educativo y las instituciones escolares buscaron adaptarse para poder mantener los procesos de aprendizaje en medio del contexto. La virtualidad, el aprendizaje remoto a distancia, se convirtieron en las expresiones "de las plataformas de comunicación, con el objetivo de llegar a la mayor cantidad de estudiantes posible" (Saavedra, 2020, s.p). fue así como en el comunicado número tres del 11 de marzo del 2020, el Centro de Coordinación Institucional de Operaciones (CCIO) de la Universidad de Costa Rica (UCR) informó a la comunidad universitaria y al personal docente suspender, a partir de ese momento, las clases presenciales e implementar una modalidad de enseñanza virtual que garantizara la continuidad y calidad. Lo anterior, como resultado de la evolución epidemiológica del COVID-19 en el país.

La manera como se puede describir la respuesta inicial que brindó la Universidad de Costa Rica ante ese escenario, debe ser explicada como espontánea, en cuanto implicó hacer uso de las plataformas y de las herramientas institucionales para la continuación del proceso de educación remota, fue así que en el comunicado número cinco con fecha del 11 de marzo del 2020, se oficializó que los cursos dentro de la institución, "[...] se desarrollarán mediante la plataforma de Mediación Virtual de la Vicerrectoría de Docencia".

A partir de este momento, la comunidad universitaria entró en un proceso de reflexión, análisis y discusión que permitió la mejor toma de decisiones en aras de la continuidad del semestre. La resolución VD11417-2020 puntualiza una serie de elementos que permitió generar una estructura de trabajo en medio de la crisis del COVID-19 a lo interno de la Universidad. Dentro de los principales puntos que se definieron en dicho documento, se destacan aspectos como: ajuste de los programas de curso para la readaptación y reprogramación de los contenidos, la flexibilización de actividades académicas utilizando las TIC y los recursos que tuvieran disponibles y pertinentes en la disciplina, la identificación detallada del 
acceso a equipos y herramientas de trabajo con tecnologías de información y comunicación (TIC) por parte del estudiantado, así como el uso prioritario y pertinente. (Universidad de Costa Rica, 2020).

En ese marco, la cátedra de Introducción a la Pedagogía, subscrita al Departamento de Educación Secundaria de la Escuela de Formación Docente en la Facultad de Educación (UCR) decidió hacer uso de la bitácora como estrategia didáctica en todos sus grupos, siendo la principal medida en el rediseño para atender la emergencia. La argumentación que justificó su empleo en el curso partió de la consideración de ser una herramienta para el desarrollo de contenidos, habilidades y objetivos propuestos en el semestre, además de ser una opción ante la variedad de escenarios en cuanto a la accesibilidad de recursos tecnológico que presentaban el conjunto de estudiantes matriculados en el curso.

Así, la bitácora se asumió como un recurso versátil, en cuanto favoreció la organización en el desarrollo de la asignatura, propició la toma de decisiones de las personas docentes, con la intención de responder a las necesidades del conjunto de estudiantes. Además de favorecer un acompañamiento directo ante la suspensión de la presencialidad en el primer semestre 2020, todo eso mediante un proceso de flexibilización del proceso de aprendizaje.

La génesis del presente artículo implicó conocer los aportes y limitaciones de la implementación de la bitácora como estrategia didáctica durante la crisis del COVID-19 y su valoración con respecto al alcance de los procesos de aprendizaje por parte del estudiantado que participa en el curso Introducción a la Pedagogía de la Universidad de Costa Rica durante el primer semestre 2020.

Para el abordaje de los referentes conceptuales, la presente investigación asumió el concepto de la bitácora, el cual recae históricamente en la navegación, los marineros tenían "[...] una especie de armario, situado junto al timón o en el puente de mando de un barco, en el que se guarda la brújula y un cuaderno, el cuaderno de bitácora, en el que el capitán debe anotar diariamente la travesía realizada ese día y las incidencias ocurridas" (Palomero y Fernández, 2005, p. 4). La intención de los marineros de llevar un registro de sus viajes tenía un doble sentido, por un lado, hacer una descripción de los sucesos acaecidos a lo largo de la travesía, en especial de cómo se resolvieron los problemas y, de esta manera, el registro de estos hechos se convertían en experiencias útiles para futuros emprendimientos. Para Vera (2015) las bitácoras son:

[...] como un diario de a bordo, como un lugar donde guardamos lo que nos orienta y guía; un lugar a dónde podemos ir cuando no sabemos a dónde estamos, a dónde vamos, es una estrategia ideada para contribuir a la formación integral (académica, personal y profesional) de los estudiantes. (p. 809)

En el contexto de la pandemia del COVID-19, la bitácora brinda un escenario de trabajo para el registro de avances en los aprendizajes, dudas de los contenidos desarrollados e inquietudes sobre los mismos.

En relación con los objetivos que se percibe con el uso didáctico de la bitácora, Palomero y Fernández (2005) plantean que a través de ella el grupo de estudiantes desarrollan la capacidad de hacer prácticas y de argumentar a partir de referentes científicos; se acostumbran al registro de observaciones, aunque sea de un modo elemental; hacen explícitos los propios dilemas; logran experiencias pedagógicas y las aprovechan para el logro de aprendizaje de contenidos relacionados con una asignatura concreta y, con ello, reflexionan sobre el curso ordinario de los acontecimientos y de la vida. Un último objetivo de la bitácora es convertir el curso en oportunidad para el crecimiento y desarrollo personal.

El curso de Introducción a la Pedagogía se implementó a partir de cuatro partes estructurantes, a saber:

1. Actividades para el análisis de las lecturas. Para su desarrollo cada docente elaboró diversidad de propuestas haciendo uso de los diferentes recursos en donde se incluyó el empleo de tecnología. Algunos ejemplos de actividades desarrolladas son: resolución de problemas, esquemas resúmenes 
(de sol, árbol de problemas, uve heurística, mapa de telaraña), estudios de casos, simulación de situaciones educativas, vídeo foro, foros de discusión de temáticas.

2. La segunda parte de la bitácora se tituló "aprendizajes logrados", donde se buscó que el grupo de estudiantes planteara una reflexión personal sobre la temática desarrollada en la clase. Dicha postura se acompañó con su respectivo sustento teórico. Con esto, se buscó el desarrollo del conocimiento como un proceso que se alcanzaría de forma progresiva a lo largo del curso.

3. El tercer apartado se estructuró como inquietudes, en el cual se pretendió evocar la parte emocional del grupo de estudiantes, ya que implicaba aquellos aspectos que le llamaban la atención en la temática.

4. Finalmente, se puntualizaron las dudas, a través de las cuales, el grupo de docentes acompañaban el proceso de aprendizaje, con ello se garantizó una atención individual, dándole seguimiento a los aspectos que no quedaron claros del tema y que, por ende, son necesarios de ser retomados o ampliados.

La figura 1 expone la estructura descrita anteriormente:

Figura 1. Orden de la bitácora

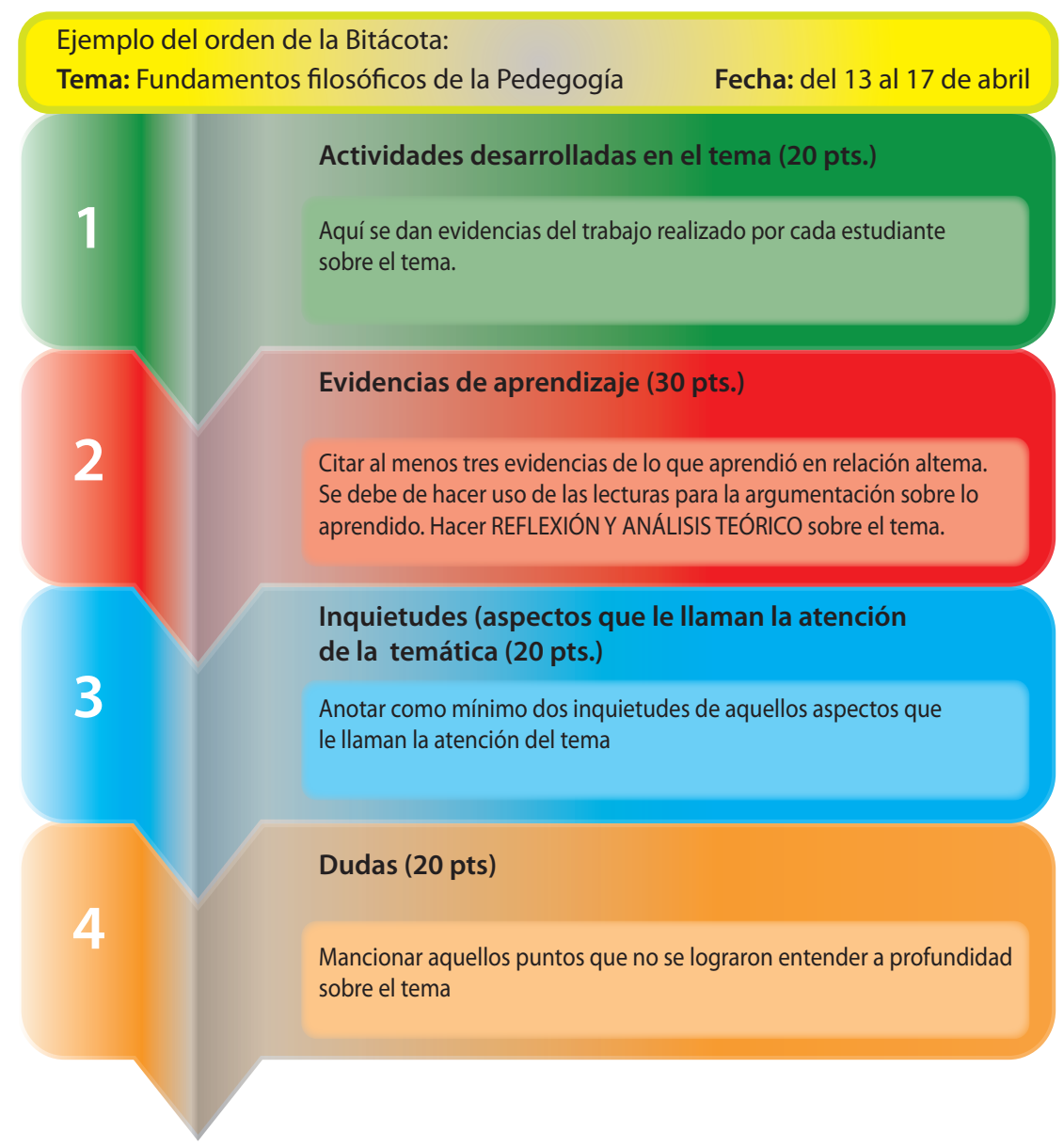

Fuente: Cátedra Introducción a la Pedagogía (2020). 
Para la presente investigación, las estrategias didácticas se definen como el conjunto de actividades organizadas cuya meta es la construcción del aprendizaje y el alcance de los objetivos planteados. Díaz (1998), citado por Flores, Ávila, Rojas, Sáez, y Díaz (2017), las conceptualiza como: “[...] procedimientos y recursos que utiliza el docente para promover aprendizajes significativos, facilitando intencionalmente un procesamiento del contenido nuevo de manera más profunda y consciente" (2017, p. 22).

Según Tapia (1997), citado por Ávila y otros (2017), las estrategias didácticas se pueden clasificar en dos tipos: aquellas enfocadas en el docente, por tanto, se centra en el proceso de enseñanza, buscan promover y facilitar el aprendizaje significativo de los estudiantes (p. 13) y aquellas dirigidas al aprendizaje, las cuales "[...] son utilizadas por el estudiante para reconocer, aprender y aplicar la información y/o contenido" (Tapia 1997, citado por Ávila y otros 2017, p. 13). Las bitácoras son usadas en esa segunda línea, ya que si bien es cierto fueron propuestas por el personal docente de la cátedra de Introducción a la Pedagogía, terminan siendo un recurso para que el grupo de estudiantes edifique su propio conocimiento.

El Diccionario de la Real Academia Española define el concepto de aporte como una "contribución, participación y ayuda" (1919). La investigación asume la categoría ligada a la bitácora como estrategia didáctica, porque en medio de la crisis provocada por el COVID-19 brinda un refuerzo en la construcción de los aprendizajes de las personas discentes en el caso específico del curso Introducción a la Pedagogía, durante el primer semestre del 2020.

La limitación es un término que alude a "acortar, ceñir" (Real Academia Española, 1919), y expone aqueIlas condiciones voluntarias o forzadas de un objeto, en este caso la bitácora como estrategia didáctica, que impide el logro de los objetivos de aprendizaje.

De esta manera, la presente investigación responde al objetivo de diagnosticar los aportes y limitaciones de la bitácora como estrategia didáctica durante la crisis del COVID-19, para su valoración en el alcance de los aprendizajes en la población estudiantil universitaria en el desarrollo del curso Introducción a la Pedagogía durante el primer semestre 2020.

\section{MATERIALES Y MÉTODOS}

El enfoque de la investigación es el cualitativo, porque "[...] describe y analiza las conductas sociales colectivas e individuales, las opiniones, los pensamientos y las percepciones" (McMillan y Schumacher, 2005 , p. 400) de los sujetos participantes acerca de un tema, en este caso, sobre las posibilidades y limitaciones de la bitácora como estrategia didáctica durante la crisis del COVID-19, así como sus aportes en el desarrollo de aprendizajes en la población estudiantil universitaria. La investigación cualitativa, "[...] recopila los datos en situaciones reales por interacción con personas seleccionadas en su propio entorno" (McMillan y Schumacher, 2005, p. 400), es así como la presente investigación se abordó en el contexto del curso de Introducción a la Pedagogía durante el primer semestre del 2020.

Dentro del enfoque cualitativo, los sujetos se convierten en agentes clave que permiten el retrato desde sus experiencias y punto de vista, Hernández, Fernández y Baptista (2010) consideran que el objetivo de los estudios cualitativos es "[...] examinar la forma en que los individuos perciben y experimentan los fenómenos que los rodean, profundizando en sus puntos de vista, interpretaciones y significados" (p. 358). De esta forma, las percepciones y visiones personales en relación con la bitácora como estrategia didáctica.

Los informantes clave correspondieron a estudiantes y docentes del curso de Introducción a la Pedagogía pertenecientes a la Escuela de Formación Docente de la Universidad de Costa Rica. El grupo de alumnos se materializó en cuatro grupos completos de los doce que conformaban la Cátedra, se trabajó con un total de 93 estudiantes: desglosados de la siguiente forma: 15 alumnos del grupo 02; 20 discentes del 
grupo 12; 30 estudiantes del grupo 07 y 30 que integraban el grupo 09. Es importante destacar que los criterios de selección se establecieron tomando en cuenta que las personas estudiantes estuvieran matriculadas y fueran participantes activos durante el desarrollo del curso y por ende que hubieran tenido la experiencia de elaborar las bitácoras como estrategia didáctica.

Se clarifica que el uso de dicha estrategia fue exclusivo del curso de Introducción a la Pedagogía como respuesta a la crisis ocasionado por el COVID-19. Por tanto, el grupo de académicos con los que se trabajó fueron quienes impartieron el curso, por ende, hicieron uso de la bitácora como estrategia didáctica.

Hernández, Fernández y Baptista (2010) señalan que la investigación cualitativa se caracteriza por un fuerte interés en las experiencias de las personas participantes, mediante la recolección de información detallada y profunda, busca conocer "[...] la diversidad de idiosincrasias únicas de los participantes inmersos en el proceso" (p. 364). Lo anterior resulta de especial interés para la investigación, puesto que las experiencias son fundamentales a la hora del análisis de las posibilidades y limitaciones de la bitácora como estrategia didáctica, de esta manera se vuelve pertinente la explicación de la selección de técnicas que permitieron la recolección de la información, la cual se sistematiza en la tabla 1:

TABLA 1

Síntesis de las referencias metodológicas de la investigación

\begin{tabular}{|c|c|c|c|c|}
\hline $\begin{array}{l}\text { Pregunta } \\
\text { problema }\end{array}$ & Objetivo general & $\begin{array}{l}\text { Objetivos } \\
\text { específicos }\end{array}$ & Categorías de análisis & $\begin{array}{c}\text { Técnicas de recolección } \\
\text { de la información de datos }\end{array}$ \\
\hline \multirow{2}{*}{$\begin{array}{l}\text { ¿Cómo determinar } \\
\text { los aportes y limita- } \\
\text { ciones de la bitáco- } \\
\text { ra como estrategia } \\
\text { didáctica durante la } \\
\text { crisis del COVID-19 } \\
\text { y su valoración } \\
\text { en alcance de los } \\
\text { aprendizajes en la } \\
\text { población estudian- } \\
\text { til universitaria del } \\
\text { curso Introducción } \\
\text { a la Pedagogía im- } \\
\text { partido el primer } \\
\text { semestre 2020? }\end{array}$} & $\begin{array}{l}\text { Diagnosticar los } \\
\text { aportes y limitacio- } \\
\text { nes de la bitácora } \\
\text { como estrategia di- } \\
\text { dáctica durante la cri- } \\
\text { sis del COVID-19, para } \\
\text { su valoración en el al- } \\
\text { cance de los aprendi- }\end{array}$ & $\begin{array}{l}\text { Reconocer los alcances y li- } \\
\text { mitaciones del uso de la bi- } \\
\text { tácora de aprendizaje como } \\
\text { estrategia didáctica durante } \\
\text { la crisis del COVID-19. }\end{array}$ & $\begin{array}{l}\text { - Aportes de la bitácora } \\
\text { como estrategia di- } \\
\text { dáctica de educación a } \\
\text { distancia. } \\
\text { - Limitaciones de la } \\
\text { bitácora como estra- } \\
\text { tegia didáctica de edu- } \\
\text { cación a distancia. }\end{array}$ & \multirow{2}{*}{$\begin{array}{l}\text { - Aplicación de dos entre- } \\
\text { vistas semiestructuradas } \\
\text { a docentes del curso de } \\
\text { Introducción a la Pedagogía. } \\
\text { - Aplicación de un cuestiona- } \\
\text { rio cualitativo a } 70 \text { estudian- } \\
\text { tes de los cuatro grupos de } \\
\text { introducción a la Pedagogía. } \\
\text { - Aplicación de tres entre- } \\
\text { vistas semiestructuradas } \\
\text { a estudiantes del curso } \\
\text { Introducción a la Pedagogía. }\end{array}$} \\
\hline & $\begin{array}{l}\text { zajes en la población } \\
\text { estudiantil universita- } \\
\text { ria en el desarrollo del } \\
\text { curso Introducción a } \\
\text { la Pedagogía duran- } \\
\text { te el primer semestre } \\
2020 \text {. }\end{array}$ & $\begin{array}{l}\text { Analizar el papel que cum- } \\
\text { plió el uso de la bitácora de } \\
\text { aprendizaje en el logro de } \\
\text { los objetivos del curso y el } \\
\text { alcance de conocimientos } \\
\text { en la población estudiantil } \\
\text { universitaria. }\end{array}$ & $\begin{array}{l}\text { - La bitácora y el alcan- } \\
\text { ce de aprendizaje en la } \\
\text { población estudiantil } \\
\text { universitaria. } \\
\text { - Logro de los objetivos } \\
\text { del curso. }\end{array}$ & \\
\hline
\end{tabular}

Fuente: Elaboración propia (2020).

La entrevista cualitativa se caracteriza por ser flexible, íntima y abierta, con el fin de intercambiar información entre el entrevistador y el entrevistado que resulte en la construcción conjunta respecto a un tema. Para fines de la investigación, la entrevista semiestructurada brindó un mayor acercamiento para el cumplimiento de los objetivos ya que se caracteriza por tener una guía de preguntas para dirigir los temas que el entrevistador requiere, pero con la libertad de poder introducir otras adicionales y así obtener más información relacionada (Hernández, Fernández y Baptista, 2010, p. 403).

Se realizaron tres entrevistas a estudiantes del curso, las cuales representaron experiencias diversas, la selección permitió tener en cuenta tres escenarios distintos que se puntualizan a continuación. La primera entrevistada fue una estudiante de primer ingreso, este caso interesaba su valoración en la poca experiencia universitaria. Por el contrario, la segunda estudiante fue seleccionada por la cantidad de años cursados en la universidad, de tal manera que su recorrido fuera clave como criterio a la hora de dar sus experiencias en relación con el objeto de estudio. La última participante fue una extranjera matriculada en el curso, cuyos aportes implicaba la valoración de la temática, pero vista desde su perspectiva. 
El cuestionario se considera un medio útil y eficaz para recoger ìnformáción en un tiempo breve. Se caracteriza por ser un proceso de investigación donde se aplica una entrevista altamente estructurada y el sujeto que responde proporciona información sobre un tema en específico (Sampieri 2010, p. 217). Esta técnica se utilizó mediante una guía de preguntas, con el fin de recolectar los datos y conocer los puntos de vista de manera individual acerca de las posibilidades y limitaciones de la estrategia didáctica y sus aportes al desarrollo de los aprendizajes en el grupo de estudiantes; al final se logró aplicar a setenta estudiantes, quienes fueron los que lograron contestarlo.

En relación con la validez, permite garantizar la rigurosidad a la investigación, Hidalgo (2005) expone que el objetivo del proceso es "[...] otorgarle a los instrumentos y a la información recabada, exactitud y consistencia necesarias" (p. 2). Para garantizar este planteamiento, cada uno de los instrumentos fue validado por expertos, los cuales dieron sus criterios en busca de retroalimentar y mejorar cada uno, de tal forma que el investigador se garantizó aplicar la mejor versión de los mismos.

A fin de organizar debidamente la información recabada por medio de las técnicas de recolección mencionadas previamente, se siguió un proceso de tres pasos. En primera instancia se transcribió cada una de la información. Posteriormente, se organizó y resumió la misma a través de las categorías de análisis. Y, por último, se analizó por medio de procesos de triangulación, análisis de discurso y análisis de contenido.

Como punto de partida, se respaldó la recolección de la información a través de grabaciones, vídeos, y con los datos conseguidos por medio de las técnicas aplicadas. Luego, se procedió a la transcripción de manera detallada y fiel a los aportes, señalamientos y comportamientos de los sujetos de investigación. Posteriormente, se construyeron matrices y gráficos para organizar la información por medio de categorías que permitieran resumirla y contrastarla con los aportes teóricos recabados para la investigación.

Por último, en pro de asegurar la credibilidad de los hallazgos alcanzados por medio de aplicación de las técnicas mencionadas, se establecieron mecanismos que aseguraron la validez a la investigación, alejándose de la simple interpretación subjetiva por parte del investigador. Según Gurdián (2007) “[...] la calidad de un estudio está determinada, en gran medida, por el rigor metodológico con que se realizó la investigación" (p. 222), por tanto se procedió al análisis mediante la triangulación, a fin de poder alcanzar la confiabilidad y credibilidad pertinentes a un trabajo de este tipo. Según Gurdián (2007), para interpretar la información recolectada, se pueden utilizar distintos tipos de triangulación. Para efectos de la presente investigación se optó por la triangulación de técnicas.

\section{DISCUSIÓN DE RESULTADOS}

El hallazgo principal del estudió permitió el reconocimiento de que la bitácora es una estrategia didáctica útil para el desarrollo del curso en medio de la crisis provocada por la pandemia. La bitácora fomentó la capacidad de síntesis y el desarrollo de un conocimiento integrador, el alcance de un aprendizaje significativo y útil para el grupo de estudiantes, el desarrollo del autoaprendizaje, la retroalimentación docente en un contexto de virtualidad, la flexibilización de los procesos de aprendizajes según las necesidades y realidades de estudiantes y docentes. La principal limitación es que su uso requiere mucho tiempo y evidencia un desgaste a lo largo del semestre, así como el acceso a Internet.

Los resultados se agruparon a partir de las categorías de análisis de la investigación a saber: aportes y limitaciones de la bitácora como estrategia didáctica, la bitácora y el alcance de aprendizaje en la población estudiantil universitaria y el logro de los objetivos del curso.

En cuanto a los portes de la bitácora como estrategia didáctica durante la crisis del COVID-19, 83,8\% del grupo de estudiantes entrevistados expresaron su grado de satisfacción en relación con el uso de la bitácora en el curso, eso queda aún más evidenciado cuando se reconoce lo siguiente: 
"[...] la técnica de la bitácora es muy interesante, si es una estrategia que nace de una crisis y por lo tanto no es perfecta, siento yo porque evidencia un desgaste a lo largo del semestre, creo que uno hace su mejor esfuerzo en cada bitácora, lo cual requiere mucho tiempo, pero fue muy interesante una metodología que al fin y al cabo salió muy bien." (Estudiante del curso Introducción a la Pedagogía, comunicación personal, jueves 02 de julio del 2020).

El resultado se complementa con lo expresado por las personas discentes en el cuestionario ante la pregunta de cómo calificaría la bitácora como estrategia didáctica para los cursos universitarios en medio de la crisis por la pandemia de COVID-19. Los resultados arrojan una calificación positiva sobre el uso de la bitácora, siendo las notas 8, 9 y 10 las de mayor valor, en donde la nota 8 obtuvo 33,8\%, el 9 (31\%) y el 10 (18,3\%). Dentro de las explicaciones que se pueden establecer a este punto de la investigación, se encuentra el reconocimiento de su utilidad.

"[...] una plataforma donde al recoger todas las actividades e integrar reflexiones respecto al tema trabajado. Este tipo de herramienta nos ayuda a tener un fácil acceso al trabajo de la asignatura y a concentrar el aprendizaje sobre un tópico concreto para su futura consulta." (Estudiante del curso Introducción a la Pedagogía, comunicación personal, miércoles 01 de julio del 2020)

Una vez que se han evidenciado los aportes de las bitácoras como estrategia didáctica por parte del grupo de estudiantes, es importante recabar, desde la mirada del grupo de informantes, aquellos aspectos puntuales de forma ordenada, de tal manera que se logró hacer una clasificación de los aportes según lo expuesto por Canalejas (2010). La tabla 2 presenta la información:

TABLA 2

Opiniones del grupo de estudiante sobre los aportes de la bitácora de aprendizaje.

\section{Aportes}

Capacidad de síntesis y conocimiento integrador

Fortalece el trabajo en equipo

"Las diferentes actividades hacen que se pueda desarrollar la capacidad de síntesis, así como hacer un análisis íntegro de la materia de cada clase." (Estudiante 1)

"Uno va haciendo como su propio resumen de la materia, se hicieron siete bitácoras y cada una es como un resumen de la materia vista en las clases." (Estudiante 2)

"Fortalece trabajo en equipo y apoyar a estudiantes de primer ingreso en su proceso de "acomodo" a la etapa Universitaria." (Estudiante 3)

"Además permite tener más acercamiento con el profesor y a él le permite observar qué tanto están haciendo una

Comunicación buena interpretación de las lecturas sus estudiantes." (Estudiante 4)

docente y estudiante

"Dentro del ámbito de educación a distancia, existe un aporte de la bitácora muy positivo, que es que el profesor pueda conocer el alcance del aprendizaje a través de las conclusiones y la reflexión." (Estudiante 5)

Fomenta la creatividad

"Permite ser creativo al momento de elaborarla, por ejemplo, en el diseño, poder incluir chistes, etc. Por lo que el proceso de creación es más llamativo o atractivo que un simple resumen, además al hacerla se presta más atención a los detalles por lo que el aprendizaje queda más profundo." (Estudiante 6)

"El trabajo en la bitácora implica que uno no solo estudia para un examen, hay veces se embota y todo el estrés porque si no se pasa el examen me voy a quedar, en cambio con todo el trabajo de la bitácora es más fácil ver lo que uno ha aprendido y es algo que no se me va a olvidar." (Estudiante 3)

"Lo mejor es que son hechas por uno mismo y le queda el respaldo para poder utilizarlo más adelante, porque fue

Aprendizaje significativo hecho por mi persona, en donde plasme los conocimientos en base a las lecturas y explicaciones en las clases y siento que fue de gran ayuda y que van a ser muy útil porque son como las bases para lo que sigue [...]." (Estudiante 7)

"Desafío en sí de tener que leer y tener que evidenciar eso que estamos aprendiendo y que lo estamos logrando." (Estudiante 8)

"Me parecieron una buena manera de ir evaluando cada semana lo aprendido, sin tener que ser necesariamente un examen." (Estudiante 5) 


\section{Aportes}

"De igual forma personalmente como estudiante, permite ser crítico respecto a su propio trabajo." (Estudiante 2)

"Las evidencias de aprendizaje permiten saber si hace que uno sepa si las lecturas se están haciendo a conciencia." (Estudiante 1)

Desarrollo de auto

"Son muy útiles para demostrar el aprendizaje adquirido." (Estudiante 3)

aprendizaje

"Nos propone la iniciativa de ser más responsables con el curso, puesto que la realización de la bitácora toma tiempo." (Estudiante 9)

"Nos ayuda a hacer un autoanálisis de sí realmente aprendimos algo del tema." (Estudiante 10)

Aprendizaje

integrador y como

"Las bitácoras también permiten dar como un seguimiento a la línea del curso, que al final se puede juntar todo y proceso

se da cuenta que todas las anteriores han servido para hacer las últimas" (Estudiante 11),

"Me gustó trabajar con ellas y aunque son complicadas uno aprende mucho, a veces uno lee y cree que entiende pero al hacer un trabajo como este realmente requiere un análisis profundo de la lectura, y de igual forma el crear

Utilidad futura. este tipo de trabajos es de mucha ayuda y no solo para ahora si no también para un futuro!" (Estudiante 2)

"Las bitácoras nos van a hacer muy útiles en el futuro como docentes, la actividad es un recurso que queda." (Estudiante 9)

Retroalimentación docente

"Muchas veces era difícil y ¡no porque entendiera todo! ¡Sino porque el pensar qué es lo que realmente uno no entiende a veces es complicado hasta de explicar!, entonces requiere de trabajo, de igual forma su compromiso para revisar y darnos una retroalimentación fue muy acertada." (Estudiante 7)

"Para mí el uso de las bitácoras fue indispensable para obtener el conocimiento que necesito, fue una manera más fácil de aprender." (Estudiante 4)

"Considero que las bitácoras fueron un buen recurso debido a la situación y una forma más ligera de ver el conte-

Flexibilización de nido." (Estudiante 1)

aprendizajes

"El uso de las bitácoras me permitió hacer más democrático el proceso de aprendizaje en medio de la diversidad de escenarios tecnológicos en el que estaban los estudiantes, puede darles opciones de que trabajaran con o sin computadora, que sacaran fotos y me las enviaran para su revisión, fue una buena salida en medio de un contexto en donde del todo no estábamos preparados." (Estudiante 2)

"Son muy buenas para evaluar de manera virtual, si se trabajan de manera ordenada por parte del docente como del estudiante." (Estudiante 4)

"Conforme se desarrolló el curso, los estudiantes sabían que tenían que hacer y cómo hacerlo, esto permitió que cada quien cumpliera su rol... yo como docente y ellos y ellas como estudiantes, la bitácora me garantizó como docente dar cierta certeza en medio de la incertidumbre inicial en donde yo mismo me cuestionaba cómo iba a desarrollar el curso y desarrollar un proceso de aprendizaje los más democrático, sin que la crisis fuera la causa para que los estudiantes se quedaran atrás [...]." (Estudiante 11)

"Me gustó las dinámicas porque hubo mucha incertidumbre cuando nos dijeron que iba a ser virtual, no se sabía cómo iban a calificar o dar los cursos, pero las bitácoras, al menos a mí, al leer el material y plasmarlo en la bitácora, hay una evidencia que les queda todo ese conocimiento." (Estudiante 12)

Fuente: elaboración propia a partir de entrevistas y cuestionario realizados a estudiantes y docentes del curso Introducción a la Pedagogía entre el 29 de junio y el 3 de julio.

El principal resultado relacionado a la categoría sobre las limitaciones de la bitácora como estrategia didáctica durante la crisis del COVID-19, desde la experiencia del personal docente y el grupo de estudiantes, gira en torno al tiempo para su ejecución, al preguntarle sobre la principal limitación un estudiante afirmó: "Tal vez el tiempo que se espera para cada actividad, en realidad uno dura más haciéndolo" (estudiante del curso Introducción a la Pedagogía, comunicación personal, miércoles 01 de julio del 2020).

Lo que se plantea es que en la relación entre el tiempo planeado por la persona docente para el desarrollo de las actividades propias de la bitácora y lo que se duraba haciéndola, siempre se llevó más de lo estipulado, lo cual provocó preocupación y desgaste por parte del grupo de estudiantes.

Aunado a lo anterior, las personas estudiantes exponen la complejidad en el trabajo en la realización de la bitácora, en donde "[...] el trabajo semanal se hace demasiado arduo. Tenemos que ver que, el trabajo semanal se compone de; lectura, comprensión y análisis de los textos, realización de las actividades" (estudiante del curso Introducción a la Pedagogía, comunicación personal, jueves 02 de julio del 2020). Las consecuencias del proceso se pueden puntualizar en las siguientes ideas: 
- Hacer la bitácora por hacerla, sin aprender a través de ella, “[...] al final, se hace denso, y no se puede evitar escribir para rellenar" (estudiante del curso Introducción a la Pedagogía, comunicación personal, miércoles 01 de julio del 2020).

- Sobredemanda de trabajo tanto para estudiante como para docentes, "[...] me ha ido bien solo que siento que son muchas, y creo que para el próximo semestre hay que cambiar un poco la evaluación porque es DEMASIADO trabajo" (docente del curso Introducción a la Pedagogía, comunicación personal, miércoles 01 de julio del 2020).

- Cansancio, "[...] pero el problema era que siempre durábamos mucho más del tiempo indicado, nos dedicábamos solo a eso, y durábamos mucho tiempo y realmente era cansado" (estudiante del curso Introducción a la Pedagogía, comunicación personal, jueves 2 de julio del 2020).

Otro de los aspectos que sobresale como limitación en la realización de las bitácoras fue el acceso a internet o recursos tecnológicos, que sin duda fue parte transversal del proceso de la presente investigación, de esta forma se plantea que "[...] lo más complicado (como toda la virtualización en general) es la limitación al acceso a Internet o la conexión de mala calidad, lo que en ocasiones generaba dificultades a la hora de entregar trabajo o estar en las explicaciones sincrónicas del trabajo para las bitácoras" (estudiante del curso Introducción a la Pedagogía, comunicación personal, viernes 3 de julio del 2020).

Otra de las limitaciones de la bitácora como estrategia didáctica se refiere a la estructura en la que se diseñó, se expone que las partes eran redundantes; es decir, lo que realizaban en un apartado y se repetía en otro, así por ejemplo: "[...]tienen puntos que quizás son repetitivos ya que algunas respuestas en las preguntas es básicamente la respuesta de evidencia de aprendizaje" (estudiante del curso Introducción a la Pedagogía, cuestionario aplicado el entre el 29 de junio y 3 de julio del 2020).

Aunado a lo anterior, se expone la monotonía, restándole valor en su desarrollo, "Siento que una bitácora todas las semanas en algo muy monótono entonces no me parece una excelente técnica didáctica" (estudiante del curso Introducción a la Pedagogía, cuestionario aplicado el entre el 29 de junio y 3 de julio del 2020).

Además, se dijo que la estructura de las bitácoras restó libertad y limitó la expresión ya que "[...] estoy acostumbrado a hacer un análisis más libre, yo me inspiro, yo me emociono, pero en ocasiones en el desarrollo de las actividades me sentía limitado a realizar lo que se nos pedía, considero que son redundantes" (estudiante del curso Introducción a la Pedagogía, comunicación personal, miércoles 01 de julio del 2020).

Finalmente, se aduce mayor necesidad de contacto humano a la hora de generar retroalimentación, lo que puede limitar un poco el aprendizaje, para ello, el grupo de estudiantes hacen propuesta como que "[...] sería importante poder exponer al menos una vez al semestre cada grupo o pareja su propia bitácora con el resto del grupo, esto puede propiciar las relaciones entre los compañeros así como aprender de otros" o "[...] tener mini reuniones personales o de cada pareja con el profesor, para tener una retroalimentación de la bitácora más específica" (estudiante del curso Introducción a la Pedagogía, comunicación personal, miércoles 01 de julio del 2020).

\section{LA BITÁCORA Y EL ALCANCE DE APRENDIZAJE EN LA POBLACIÓN ESTUDIANTIL UNIVERSITARIA}

Medular en el análisis es la referencia a que la bitácora permite el alcance del aprendizaje en el grupo de estudiantes, por ende, el logro de los objetivos del curso. Tal y como la afirma la docente del curso: 
Considero que las bitácoras fueron una estrategia didáctica efectiva debido a que contribuyeron con el logro de los objetivos del curso. Brindaron un espacio para que los y las estudiantes pudieran opinar sobre los contenidos del curso y analizarlos con base en lecturas que se trabajaron a lo largo del semestre. (Docente del curso Introducción a la Pedagogía, comunicación personal, jueves 2 de julio del 2020)

En medio de la crisis originada por el desarrollo de la pandemia del COVID-19 en la sociedad costarricense, la implementación de la bitácora se convirtió en una opción que permitió desarrollar el curso y el logro de sus objetivos, al tiempo que propicio el desarrollo de aprendizajes y habilidades en el grupo de estudiantes.

En cuanto a si la bitácora como estrategia didáctica permite el alcance de los aprendizajes, los resultados dan una respuesta afirmativa ya que $42,9 \%$ del grupo de estudiantes estuvieron muy de acuerdo, y $47,1 \%$ contestaron que están de acuerdo lo cual suma $90 \%$ de las respuestas. Lo anterior se contrasta con lo expresado por un estudiante del curso de Introducción a la Pedagogía cuando afirma "[...] para mí el uso de las bitácoras fue indispensable para obtener el conocimiento que necesito, fue una manera más fácil de aprender" (estudiante del curso Introducción a la Pedagogía, cuestionario aplicado el entre el 29 de junio y 3 de julio del 2020), esto coincide con lo expuesto por Murillo (2012, p.4).

En el marco de esta categoría de análisis, se hizo la consulta de si el uso de la bitácora en el curso permitió el desarrollo de habilidades como la síntesis, la argumentación y la aplicación de teorías pedagógicas, los resultados de las respuestas puntualizan que $41,4 \%$ expresaron estar muy de acuerdo con el criterio, si a eso se le suma $38,6 \%$ que respondió estar de acuerdo, el total de respuesta afirmativas suma $80 \%$, dato relevante en relación con el logro de aprendizajes a través del empleo de las bitácoras.

En relación con los resultados sobre el papel de la bitácora como estrategia didáctica y el logro de los objetivos del curso, se parte de una afirmación de un estudiante del curso: “Considero que la bitácora es un gran instrumento para alcanzar los objetivos del curso" (cuestionario aplicado entre el 29 de junio y 3 de julio del 2020), con la cita se da una idea de los resultados encontrados en esta categoría de análisis. Dicha información adquiere complemento con lo que expresan los participantes en el cuestionario ya que se evidencia como 51,4\% del conjunto de encuestados afirmaron estar de acuerdo con la bitácora como un recurso didáctico útil que les permitió desarrollar el curso, logrando sus objetivos en medio de la crisis por la pandemia de COVID-19, si a eso se le suma $38,6 \%$ que dijo estar muy de acuerdo, el resultado suma $90 \%$.

Ligado a lo anterior, en una de las entrevistas una estudiante logra entablar una relación entre el trabajo realizado con las bitácoras con el cumplimento de cada uno de los objetivos del curso, la forma como la informante va hablando y haciendo referencia al curso, evidencia el aprendizaje alcanzado, se clarifica las temáticas del curso, y el desarrollo de habilidades como la comprensión de lecturas, la reflexión de temáticas propias del curso, la comparación y la aplicación de conocimientos.

Existen lecturas clave que dejan una reflexión sobre los desafíos de la docencia, además que permiten entender que el docente puede hacer mucho más que solo dar una clase. No solo se conocen las bases filosóficas, sino que se puede hacer una comparación de cómo la percepción de la pedagogía en cada período ha afectado la forma de educar e inclusive el fin.

Las primeras dos bitácoras sin duda permiten establecer relaciones entre la Pedagogía como ciencia y el desarrollo y hasta mejoramiento de las sociedades.

Las bitácoras permiten que el aprendizaje se fundamente de una forma individual, teórica, y para la situación que estamos atravesando ha logrado cumplir con los objetivos del curso. (Estudiante del curso Introducción a la Pedagogía, comunicación personal, miércoles 01 de julio del 2020) 
Lo anterior coincide con lo expuesto por Murillo en cuanto expone a la bitácora como una "[...] herramienta que pretende recopilar la información que demuestre las habilidades y logros de quienes lo realizan, su manera de pensar, de cuestionarse y cuestionar, de analizar y analizarse, de sintetizar, de producir, de crear" (Murillo, 2012, p. 20).

Desde la perspectiva docente, la bitácora fue un excelente recurso en medio de la situación de la pandemia. Logró dar un acompañamiento lo más cercano posible, no tanto para sustituir la presencialidad, pero sí para estar lo más cercano y pendiente del trabajo de los estudiantes, tal y como lo expone (Barrios, Ruiz y González, 2012):

[...] la bitácora es una forma de comunicación con el estudiante en la que se involucra como parte activa de su propio proceso de formación; su gestión es sencilla, complementa las actividades presenciales, favorece la interacción docente-estudiante y proporciona soporte a las actividades de evaluación y retroalimentación. (p. 405)

La estructura de uno de los apartados permitió que los discentes expresaran sus opiniones sobre los temas del curso, además de puntualizar sus dudas; con ello brindaron la oportunidad al docente de responder a las mismas. En ese sentido, se da una retroalimentación del conocimiento de forma directa.

[...] la bitácora me ayudó a recibir retroalimentación inmediata de los contenidos del curso. De esta forma, pude ir viendo conforme avanzaba el curso las dudas más generalizadas que existían, así como algunas reflexiones y aportes particulares de los estudiantes que pude usar en clase. (Docente del curso Introducción a la Pedagogía, comunicación personal, viernes 3 de julio del 2020)

Otro aporte de la bitácora es que fue un recurso que permitió la propia reflexión sobre el trabajo realizado por parte del estudiante, "[...] una vez que finalizaban las actividades planeadas para el desarrollo del tema, cada estudiante debía hacer un análisis personal sobre lo aprendido. Esto debía desarrollarlo con argumentación teórica, generando un proceso de autogestión sobre el propio proceso de aprendizaje" (Docente del curso Introducción a la Pedagogía, comunicación personal, viernes 3 de julio del 2020).

La autogestión permitió desarrollar los aprendizajes como un proceso, facilitó que el grupo de estudiantes relacionaran los contenidos del curso los unos con los otros, retomaran lo aprendido en los temas anteriores y los usaran como base para el logro del nuevo conocimiento, en ese sentido se define una progresividad en su desarrollo. Además de ser un recurso que permite:

[...] aclarar dudas y estar pendiente de los estudiantes. Prácticamente todas las semanas había entrega de bitácora, entonces yo estaba en constante revisión de quién entregaba y quién faltó, de esta manera consultaba qué estaba pasando. Además de que verificaba si se estaba aprendiendo o no en el desarrollo de las clases. (Docente del curso Introducción a la Pedagogía, comunicación personal, viernes 3 de julio del 2020)

Como estrategia didáctica, la bitácora ayudó a los estudiantes a "[...] hacer visible su pensamiento y a condensar la información del curso de una manera personal" (Docente del curso Introducción a la Pedagogía, comunicación personal, viernes 3 de julio del 2020). Lo anterior, relaciona a la bitácora con el constructivismo, en el sentido de que facilita o posibilita la construcción personal de conocimiento por parte del estudiantado, "[...] ya que pude obtener un panorama de las construcciones conceptuales que estaban creando los estudiantes a partir de las lecturas y las actividades de mediación propuestas" (Docente del curso Introducción a la Pedagogía, comunicación personal, jueves 2 de julio del 2020). 


\section{CONCLUSIONES}

En cuanto al objetivo sobre el reconocimiento de los alcances y limitaciones del uso de la bitácora de aprendizaje como estrategia didáctica durante la crisis del COVID-19, se pueden puntualizar las siguientes conclusiones. En primera instancia, se reconoce por parte del personal académico y el grupo de estudiantes que la bitácora es una estrategia válida que permitió desarrollar el curso de Introducción a la Pedagogía durante el primer semestre del año 2020. Se convirtió en una estrategia didáctica que permitió resolver las necesidades que surgieron a raíz de una pandemia que no se esperaba. Solventó una mediación entre el docente y sus estudiantes, además, a partir de un contexto de incertidumbre, logró desarrollar una plataforma de trabajo que al final del semestre alcanzó los aprendizajes propios del curso.

Evidencia un grado de satisfacción tanto en docentes como en estudiantes hasta el punto de que ante la pregunta de cuánto recomendaría el uso de la bitácora como estrategia didáctica para la educación a distancia universitaria durante la crisis del COVID-19, los resultados en una escala del cero al diez fueron el ocho, el nueve y el diez las mejores calificaciones con $28,6 \%$ el ocho y nueve, mientras que el diez presentó $24,3 \%$ de las respuestas.

Central en estas conclusiones es precisar los aportes del uso de la bitácora como estrategia didáctica, tal y como se anota en la tabla 2, y que se retoma a modo de evidenciarlos. Entre los principales aportes están que la bitácora permitió la capacidad de síntesis y el desarrollo de un conocimiento integrador, el alcance de un aprendizaje significativo y útil para el grupo de estudiantes, el desarrollo del autoaprendizaje, la retroalimentación docente en un contexto de virtualidad y la flexibilización de los procesos de aprendizajes según las necesidades y realidades de los estudiantes y los docentes.

Finalmente, se rescata los que se había expuesto de la bitácora desde sus orígenes en la navegación, en cuanto ser un registro que favoreció la toma de decisiones y una guía del curso, garantizando una mayor claridad en las estrategias de mediación docente y su respectiva evaluación.

En relación con las limitaciones del uso de la bitácora, se puede puntualizar la falta de tiempo como el principal aspecto que tanto docentes como estudiantes expusieron. Esto provocó un desgaste del proceso educativo manifestado en monotonía, cansancio y pérdida de sentido de las actividades por realizar.

El análisis sobre las limitaciones del uso de la bitácora no se relaciona propiamente con su apreciación como estrategia didáctica, sino con elementos que configuran el contexto general desde donde se enmarca la situación del país en cuanto a la pandemia. Es decir, la falta de tiempo y el cansancio que se le aduce al trabajo de la bitácora, deberían ser analizados como parte de una falta de preparación. Lo anterior, aunado a medidas de distanciamiento social, teletrabajo, educación virtual en toda la Universidad y por ende en todos los cursos, son insumos que, sin duda, deben ser tomados en cuenta en el análisis que expone los resultados de la investigación, y que abre el espacio a nuevos objetos de estudio.

En cuanto al segundo objetivo, sobre el análisis del papel que cumplió el uso de la bitácora de aprendizaje en el logro de los objetivos del curso y el alcance de conocimientos en la población estudiantil universitaria, se puede concluir de manera categórica y contundente que es un buen recurso para el uso en una educación virtual en medio de un escenario de crisis como el de la pandemia provocada por el COVID-19. Los resultados de la investigación son claros tomando en cuenta la valoración positiva sobre el uso de la bitácora del grupo de estudiantes y docentes y de cómo se logró concluir el curso con los mejores resultados posibles en cuanto al aprendizaje y al cumplimiento de los objetivos propuestos.

Lo anterior no niega, ni contradice, la posibilidad de exponer una serie de recomendaciones que permitan la futura implementación de las bitácoras como estrategia didáctica, entre las principales están: la 
necesidad de dosificar la cantidad de bitácoras en el curso, de tal manera que no se llegue a la saturación y agotamiento tanto de docentes como estudiantes por su uso.

Es importante revisar la estructura en cuanto a cómo se integran, se pueden eliminar partes para que la información que expone el grupo de estudiantes no sea repetitiva. Como consecuencia de lo anterior, se debe realizar una revisión de todos los componentes de la evaluación del curso, para darle un valor significativo a las bitácoras ya que se ha comprobado que son un insumo que por sí solas permiten verificar el logro de aprendizajes, pero cada una lleva un grado de complejidad, digno de ser reconocido.

El punto de vista del grupo de estudiantes que también expresaron que no les gustó el trabajo en bitácoras, es un punto que no se debe obviar, esta divergencia es valiosa en cuanto hace pensar que las estrategias de mediación obedecen a gustos y a formas de aprender. Lo valioso en este punto sería que el docente pueda proponer a través de las bitácoras recursos lo más diversos posibles, pueden incluir el uso de la tecnología, actividades análogas y digitales para que el estudiante tenga una experiencia mucho más enriquecida. Con esto se podría evitar ser una propuesta que coarte al estudiante tal y como se expresó: "[...] en el desarrollo de las actividades me sentía limitado a realizar lo que si se me pedía" (estudiante del curso Introducción a la Pedagogía, cuestionario aplicado entre el 29 de junio y 3 de julio del 2020).

Por otro lado, la presente investigación no ha pretendido generalizar en cuanto a los aportes y limitaciones de la bitácora como estrategia didáctica y su papel en el alcance de aprendizajes y objetivos del curso. Sin embargo, el diagnóstico que se ha logrado visualiza los puntos de vistas de docentes y estudiantes, se permite una aproximación real tomando en cuenta sus experiencias y puntos de vista. Esto ha facilitado, tal y como lo hacían los marineros en la antigüedad, hacer un registro de acontecimientos acaecidos a lo largo del primer semestre del año 2020, en medio de una realidad social que sin duda es histórica. Es así como esta investigación se convierte por sí misma en una bitácora de aprendizaje, en un dictamen de cómo se intentó resolver los retos en medio de una pandemia, es una evidencia de las experiencias, sentimientos y sensaciones en medio de una crisis, con la esperanza de que todo lo anterior propicie nuevas reflexiones sobre recursos didácticos exitosos empleados en medios de la pandemia, otra línea de investigación implica la reflexión acerca de si este tipo de circunstancias son una crisis o una posibilidad de cambio. Otra temática de investigación implica la reflexión sobre aquellas prácticas emergentes de innovación didáctica en contextos de crisis.

Finalmente, se debe rescatar la importancia de estudios relacionados en aras de tomar decisiones con la rigurosidad de un saber científico y así entablar procesos que promuevan mejorar el acto educativo en medio del presente escenario. Las investigaciones son espacios de discusión que, desde la academia, constatan los esfuerzos que se han hecho para disminuir el impacto negativo del COVID-19 en la educación superior costarricense. 


\section{REFERENCIAS}

Barrios, P., Ruiz, L. A. y González, K. (abril, 2012) La bitácora como instrumento para seguimiento y evaluación. Formación de residentes en el programa de Oftalmología. Revista de Investigaciones Andina, 14(24). Recuperado de https://www.redalyc.org/pdf/2390/239024334004.pdf

Canalejas, M.C. (marzo, 2010). El portafolio como herramienta didáctica: un estudio en escuelas universitarias de enfermería. EDUC MED, 13(1). Recuperado de http://scielo.isciii.es/pdf/edu/v13n1/ original4.pdf

Flores, J., Ávila, J., Rojas, C., Sáez, F., Acosta, R. y Díaz, C. (2017). Estrategias didácticas para el aprendizaje significativo en contextos universitarios. Concepción, Chile: Trama Impresores S.A.

Gurdián, A. (2007). El Paradigma Cualitativo en la Investigación Socio-Educativa. San José, Costa Rica: Investigación y Desarrollo educativo Regional (IDER).

Hernández, S., Fernández, C. y Baptista, L. (2010). Metodología de la investigación. Madrid, España: McGrawHill.

Hidalgo, L. (2005). Confiabilidad y Validez en el Contexto de la Investigación y Evaluación Cualitativas. [PDF]. Recuperado de www.ucv.ve/uploads/media/Hidalgo2005.pdf

McMillan, J. y Schumacher, S. (2005). Investigación Educativa. Madrid, España: Pearson Education.

Murillo, G. (febrero, 2012). El portafolio como instrumento clave para la evaluación en educación superior. Actualidades investigativas en educación 12(1). Recuperado de https://www.redalyc.org/ pdf/447/44723363015.pdf

Palomero, J. y Fernández, M. (febrero, 2005). El cuaderno de bitácora: reflexiones al Hilo al Hilo Del Espacio Europeo de la Educación Superior. Revista Electrónica Interuniversitaria de Formación del Profesorado, 8(4). Recuperado de https://dialnet.unirioja.es/descarga/articulo/2782457.pdf.

Real Academia Española. (1919). Definición de aporte. Diccionario en línea. Recuperado de https://dle. rae.es/aporte? $\mathrm{m}=$ form.

Real Academia Española. (1919). Definición de limitación. Diccionario en línea. Recuperado de https:// dle.rae.es/limitar?m=form

Saavedra, J. (30 de marzo de 2020). COVID-19 y Educación: Algunos desafíos y oportunidades [Mensaje en un blog]. Recuperado de https://blogs.worldbank.org/es/education/ educational-challenges-and-opportunities-covid-19-pandemic

Universidad de Costa Rica, Catedra de Introducción a la Pedagogía. (I Semestre 2020). Criterio de la Evaluación de la bitácora de Aprendizaje.

Universidad de Costa Rica, Oficina de Divulgación e Información. (11 de marzo de 2020). Comunicado 3: La UCR solicita a sus docentes implementar clases bajo una modalidad virtual [Comunicado en línea]. Recuperado de https://www.ucr.ac.cr/medios/documentos/2020/comunicado-3.pdf

Universidad de Costa Rica, Oficina de Divulgación e Información. (11 de marzo de 2020). Comunicado 5: Uso de la plataforma de Mediación Virtual de la UCR [Comunicado en línea]. Recuperado de https://www.ucr.ac.cr/medios/documentos/2020/comunicado-5-.pdf

Universidad de Costa Rica, Vicerrectoría de Docencia. (11 de marzo de 2020). Resolución VD-11417-2020: Sobre el desarrollo de la docencia mediada con tecnologías de la información y comunicación [Comunicado en línea]. Recuperado de http://vd.ucr.ac.cr/documento/resolucion-vd-11417-2020/

Vera, L. (2015). La bitácora, una estrategia didáctica que desarrolla las competencias de los estudiantes del siglo XXI. En J. A. Hernanz y M. Watty (Eds.), Tendencias y desafíos en la innovación educativa: un debate abierto (pp. 807-815). Recuperado de https://www.uv.mx/dgdaie/files/2016/10/E-bookTDIE.pdf 